\title{
Preparation and Evaluation of Solid Composites Containing Choline Alphoscerate
}

\author{
Hoon Kim* and Seong-Wan Cho ${ }^{\dagger ; *}$ \\ Department of Pharmaceutics \& Biotechnology, Konyang University, Daejeon 35365, Korea
}

\begin{abstract}
The objective of this study was to evaluate the characteristics of the mixtures of choline alphoscerate (alpha-glycerylphosphorylcholine, alpha-GPC), in the liquid form, and sucrose ester, which formed a solid composite. The choline alphoscerate solid composites were prepared using different ratios of sucrose ester, and different preparation methods, such as air drying and rotary evaporation, were compared for their preparation efficacy. We examined the characteristics of the solid composites by using scanning electron microscopy (SEM), angle of repose, and moisture content. The ideal mixing ratio of choline alphoscerate and sucrose ester was determined as 1:3 and air drying was found to be more suitable for the preparation of solid composites than rotary evaporation. SEM measurements of the degree of dispersion and the size of particles indicated that a high-temperature air method was more suitable. These results demonstrated the successful preparation of choline alphoscerate solid composites that have potential for industrial use.
\end{abstract}

Key Words: Choline, Alphoscerate, Sucrose ester, Solid composites

\section{INTRODUCTION}

Dementia is a disease characterized by a cluster of symptoms and signs manifested as difficulties in memory, disturbances in language, psychological changes, and impairment in activities of daily living. Much of the clinical research into the development of cholinergic agents has followed since their initial proposal and although the overall clinical effects remain limited, the cognitive enhancers used to modulate cholinergic function remain the most widely used medications approved for the treatment of dementia (Cummings and Benson, 1984; Grossman et al., 2006; Kidd, 2008).

Choline alphoscerate (CA; $\mathrm{C}_{8} \mathrm{H}_{20} \mathrm{NO}_{6} \mathrm{P}$, MW 257.2, CAS 28319-77-9, Fig. 1) has been known by many different names, including choline alfoscerate, glycerophosphocholine, L-erglycerylphosphorylcholine, and L-phglycerophosphorylcholine, and has been used in the treatment of Alzheimere treatase and other forms of dementia (Moreno, 2003; Parnetti et al., 2007). In addition, CA is a biosynthetic precursor of the neurotransmitter acetylcholine, and is rapidly metabolized to choline and transported across the blood-brain barrier. After oral administration, CA is absorbed via a mediated transport in the small intestine (Sheard et al., 1986; Sheard and Zeisel, 1986; Fossati et al., 1994; Park et al., 2015). After absorption, CA is rapidly cleaved by glycerylphosphorylcholine diesterase to glycerophosphate and choline, the main metabolite, which leads to blood levels of choline greater than normal (background) levels. The increased levels of choline are generally found to reach to normal levels between

Received: February 21, 2019 / Revised: March 28, 2019 / Accepted: April 15, 2019

*Professor.

†Corresponding author: Seong-Wan Cho. Department of Pharmaceutics \& Biotechnology, Konyang University, 158 Gwanjeodong-ro, Seo-gu, Daejeon 35365, Korea.

Tel: +82-42-600-8507, Fax: +82-10-9203-2061, e-mail: swcho@konyang.ac.kr

(C)The Korean Society for Biomedical Laboratory Sciences. All rights reserved.

(c) This is an Open Access article distributed under the terms of the Creative Commons Attribution Non-Commercial License (http://creativecommons.org/licenses/by-nc/3.0/) which permits unrestricted non-commercial use, distribution, and reproduction in any medium, provided the original work is properly cited. 


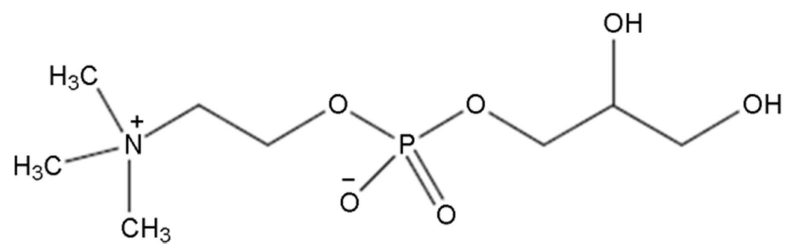

Fig. 1. Structure of choline alphoscerate (CA).

6 hours and 10 hours after oral administration (Gatti et al., 1992; Trainia et al., 2013). However, despite the advantages of CA, it has the drawback that solid forms are difficult to prepare because it is a liquid.

In this study, we aimed to increase the compliance of the liquid form of CA by using various excipients. The sucrose ester used in this experiment for the solidification of CA is a compound obtained through the esterification of some of $8-\mathrm{OH}$ of a sugar with long fatty acids of 10 to 20 carbon atoms, and is a high value-added product that can be used as a food additive with various uses, including as a surfactant, a bio-surfactant, a biodegradable surfactant absorbent, a soluble fat, or an alternative fat (Majahabulin, 2008).

Owing to the sticky nature of CA, the currently available CA products are mostly soft capsule preparations and the scope of their formulations is limited. Soft capsules are generally more susceptible to contamination than other formulations, such as powders or tablets, and the production cost of soft capsules is relatively high because of the additional processing and manufacturing equipment required. Therefore, we prepared liquid CA as a solid formulation by mixing with sucrose esters to overcome the inherent disadvantages of CA and evaluated the characteristics of the composites.

\section{MATERIALS AND METHODS}

\section{Materials and instruments}

Choline alphoscerate (DONGWOO, Korea), sucrose ester series (RYOTO, Japan), ethanol 95\% (SAMCHUN, Korea), tetrahydrofuran (SAMCHUN, Korea), 2,2,4-trimethylpentane (SAMCHUN, Korea), and all other chemicals were of analytical grade and used without further purification. In the experiments, the following instruments were used: drying oven (HB-502S, Hanback, Korea), UV/vis spectrophotom- eter (Thermo Fisher Scientific, USA), rotary evaporator (N-1000S-W, EYELA, Japan), scanning electron microscope (SEM; Hitachi, Japan), and moisture analyzer (MB45, OHAUS, Korea).

\section{Preparation and drying of CA-sucrose ester composite}

The CA-sucrose ester composite was mixed with distilled water to make a solution of CA. Four types of sucrose ester $(170,570,1,170$, and 1,670), which have different characteristics, were used. The ratios of CA-sucrose ester tested were $2: 1,1: 1,1: 2,1: 3$, and 1:4. The characteristics of the prepared complexes were evaluated by using various methods. In this experiment, two different drying methods were used and the composites were evaluated. The first method used an air dryer at 70 were two differentthe second method was distillation and rotary evaporation at 70t 7ry evaporation two different drying $\mathrm{m}$ they were investigated under controlled conditions.

\section{Angle of repose}

The angle of repose of a granular material is the steepest angle of descent or dip relative to the horizontal plane at which a material can be piled without slumping. At this angle, the material on the slope face is on the verge of sliding. One gram of each of the prepared CA-sucrose esters composites was tested. The sediment angle and angle of repose spilling out from the mouth of the triangular funnel at a height of $1 \mathrm{~cm}$ were measured. The radius and height of the deposited powder were measured by using the following equation (1):

$$
\tan \theta=\mathrm{h} / \mathrm{r}
$$

\section{Measurement of moisture content}

The moisture content is the quantity of water contained in a material. Moisture measurement is important because it is likely that $\mathrm{CA}$ will become liquid again over time if the solid composites contain a lot of water. The moisture content of CA-sucrose ester composites was measured by using a moisture meter and the weight loss was measured after the composite was held at 105 ite was held t loss was measured. 


\section{Scanning electron microscope (SEM) observation of composites}

Microscopy is the technical field of using microscopes to view objects and areas of objects that cannot be seen with the naked eye. The inspection of surface roughness represents an important part of the composite properties. The SEM was used to identify the surface properties of the CA-sucrose ester composites. The measurement voltage was $5 \mathrm{kV}$ and the surface of the composite was coated with a $10 \mathrm{~mm}$ platinum alloy by using an ion evaporator and observed using SEM.

\section{Validation of analysis method specificity}

Specificity is the ability to assess unequivocally the analyte in the presence of components that are expected to be present, despite the presence of impurities. We checked the specificity to verify the validity of the content test and the method of analysis for CA. The maximum absorption wavelength of the CA standard and sample was measured and compared to determine whether CA could be accurately measured at the determined maximum absorption wavelength without being influenced by other excipients.

\section{Validation of linearity range of analysis method}

Linearity is defined as the ability within a given range to obtain test results that are directly proportional to the concentration amount of analyte in the sample. CA $(50 \mathrm{mg})$ was dissolved in $100 \mathrm{~mL}$ of standard stock solution, and the standard stock solution was serially diluted with distilled water to prepare five standard solutions: 1,000, 500, 250, 125, and $62.5 \mathrm{ppm}$. The absorbance values for each concentration of standard solution at $254 \mathrm{~nm}$, which was the maximum absorption wavelength, were measured to obtain the straight correlation coefficient, $\mathrm{R}^{2}$. The correlation coefficient was important to verify the linearity of the CA.

\section{Validation of detection and quantitative limit of analysis method}

The detection limit of an individual analytical procedure is the lowest amount of analyte in a sample that can be detected, but not necessarily quantitated as an exact value.
The limit of detection (LOD) is the point at which a measured value is greater than the uncertainty associated with it; it is the lowest concentration of analyte in a sample that can be detected but not necessarily quantified. The limit of quantitation (LOQ) of an individual analytical procedure represents the lowest amount of analyte in a sample that can be quantitated with suitable precision and accuracy. The limit of quantitation is a parameter of the quantitative assays for low levels of compounds in sample matrices, and is used especially for the determination of impurities or degradation products. Based on the standard deviation (es or degradation products.trices, andt from the calibration curve obtained from the linearity test, the detection limit and the quantitation limit were calculated from the following equations $(2,3)$ :

$$
\begin{aligned}
& \text { Limit of detection }(\mathrm{LOD})=3.3 \sigma / \mathrm{s} \\
& \text { Limit of quantitation }(\mathrm{LOQ})=10 \sigma / \mathrm{s}
\end{aligned}
$$

\section{Evaluation of Ca content of the composites}

To measure the CA content in the CA-sucrose ester composites, $10 \mathrm{mg}$ of the composite was dissolved in distilled water, made up to a volume of $10 \mathrm{~mL}$, and then diluted 10 times with distilled water. The CA content of the diluted solution was determined by the substitution of the absorbance value measured by using a spectrophotometer into the prepared calibration curve.

\section{RESULTS}

\section{Preparation of Ca-sucrose ester composites}

Sucrose ester 170 composites did not have the ability to mix CA and sucrose powder and they separated in a similar way to oil and water. Sucrose 1,670 composites showed a tendency towards separation of the parts over time after mixing with CA. Finally, the mixtures selected were the sucrose ester 570 and sucrose 1,170 composites. After further experiments, the ratio of CA to sucrose ester ratio was determined as 1:3, which provided the most suitable stickiness and degree of liquefaction.

\section{Angle of repose}

When bulk granular materials are poured onto a horizontal 
Table 1. Angle of repose of composites

\begin{tabular}{llc}
\hline \hline Product & \multicolumn{1}{c}{ Curing method } & Angle of repose (\%) \\
\hline Sucrose & Air drying & 0.25 \\
ester-570 & Rotary evaporation & 0.18 \\
\hline Sucrose & Air drying & 0.12 \\
ester-1170 & Rotary evaporation & 0.11 \\
\hline
\end{tabular}

Table 2. Result of moisture measurement in the CA composites

\begin{tabular}{llc}
\hline \hline \multicolumn{1}{c}{ Composites } & \multicolumn{1}{c}{$\begin{array}{c}\text { Preparation } \\
\text { method }\end{array}$} & $\begin{array}{c}\text { Moisture content } \\
(\%)\end{array}$ \\
\hline Sucrose & Air drying & 1.72 \\
ester-570 & Rotary evaporation & 3.92 \\
\hline Sucrose & Air drying & 2.17 \\
ester-1170 & Rotary evaporation & 2.34 \\
\hline
\end{tabular}

surface, a conical pile will form. The internal angle between the surface of the pile and the horizontal surface is known as the angle of repose and is related to the density, surface area, and shape of the particles, and the coefficient of friction of the material. Materials with a low angle of repose form flatter piles than materials with a high angle of repose. The angle of repose of sucrose ester 570 composites was higher, regardless of the drying method (Table 1).

\section{Measurement of moisture content}

CA-sucrose ester composites were prepared by air drying and rotary evaporation and the moisture contents were compared. When prepared by hot air drying, the moisture content of the CA-sucrose ester 570 composite was $1.72 \%$ and the moisture content of the CA-sucrose ester 1,170 composite was $2.17 \%$. The water content of the composites prepared by rotary evaporation was $3.92 \%$ for the CA-sucrose ester 570 composite and $2.34 \%$ for the CA-sucrose ester 1,170 composite (Table 2). Drying condition was therefore shown to affect the properties of composites. The moisture content of the composites prepared by air drying was lower than those prepared by rotary evaporation. The initial moisture content of CA was $14 \%$. When the water content of the complex was mixed with the sucrose ester, it was confirmed that the moisture content was decreased by eight times or more, which meant that it successfully solidified.

\section{Validation of analysis method specificity}

Specificity provides an exact result of the determination of the content or potency of the analyte, and is demonstrated by the ability to discriminate between compounds of closely related structures, or by comparison with known reference materials. To validate the specificity of the method, the analysis results of the sample, consisting of the excipient (except for the CA standard solution) and composite solution were compared. As the main peak of CA was not affected, these results showed that the specificity was confirmed.

\section{Validation of linearity range of analysis method}

Linearity is the ability of the assay to return values that are directly proportional to the concentration of the analyte in the sample. To promote linearity, mathematical data transformations may be allowed if there is scientific evidence that the transformation is appropriate for the method. In this experiment, $100 \mathrm{mg} \mathrm{CA}$ was dissolved in $100 \mathrm{~mL}$ of standard solution to prepare stock solution. This standard stock solution was diluted with serial dilutions. The concentration of the final five standard solutions was 1,000, 500, 250,125 , and $62.5 \mathrm{ppm}$. The five different concentrations of CA solutions were analyzed at $254 \mathrm{~nm}$ by using a spectrophotometer. The calibration curves were plotted with the $\mathrm{x}$-axis as the concentration and the $\mathrm{y}$-axis as the absorbance. The calibration curve was $y=0.0009 x+0.0043$ and the $R h$ value was 0.9995 . The results confirmed that the CA solution was linear for the samples tested within a given range (Fig. 2).

\section{Validation of dection and quantitative limit of analysis method}

The limit of detection and the limit of quantitation for $\mathrm{CA}$ were obtained from the standard deviation of the linearity calibration curve. At a wavelength of $254 \mathrm{~nm}$, the detection limit was $1.1 \mathrm{ppm}$, which was $0.8 \%$ of the reference concentration of $125 \mathrm{ppm}$, and the quantitation limit was $3.33 \mathrm{ppm}$, which was $2.6 \%$ of the reference concentration (Table 3 ).

\section{Evaluation of CA content of the composites}

The theoretical content of CA in $1 \mathrm{~g}$ of the prepared 


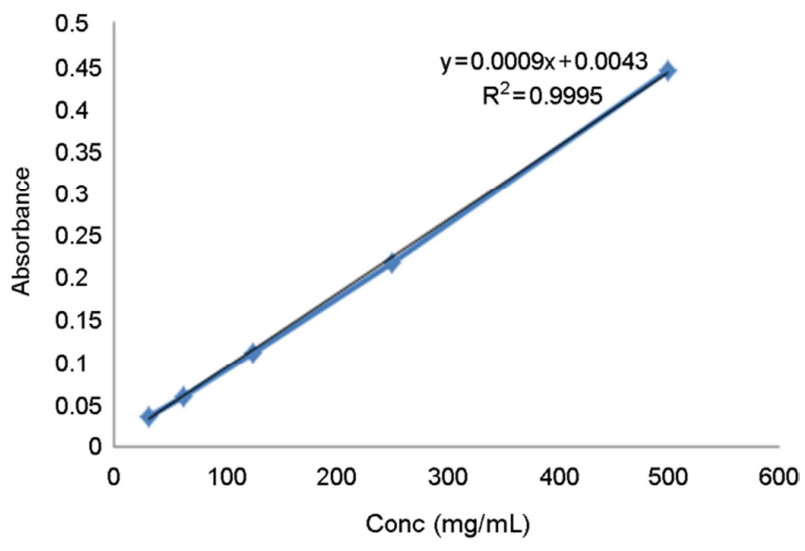

Fig. 2. Calibration curve for choline alphoscerate (CA) (X axis: concentration, $\mathrm{Y}$ axis: Absorbance).

Table 3. Results of limit of detection (LOD) and limit of quantitation (LOQ)

\begin{tabular}{ccc}
\hline \hline Wavelength $(\mathrm{nm})$ & LOD & LOQ \\
\hline 254 & 1.1 & 3.33 \\
\hline
\end{tabular}

Table 4. Result of the CA content

\begin{tabular}{llc}
\hline \hline Composites & Preparation method & Content $(\%)$ \\
\hline Sucrose & Air drying & 97.11 \\
ester-570 & Rotary evaporation & 104.89 \\
\hline Sucrose & Air drying & 96 \\
ester-1170 & Rotary evaporation & 102.67 \\
\hline
\end{tabular}

composite is $0.25 \mathrm{~g}$. The results of the CA content evaluation in the composites showed that the yield of CA in the CAsucrose ester 1,170 and 570 composites produced by rotary evaporation and air drying method was more than $95 \%$. These results showed that the composites were well prepared and characterized (Table 4).

\section{SEM of CA-sucrose composites}

An SEM is a type of electron microscope that scans the surface of a sample with a focused beam of electrons to produce an image of the same. The electrons interact with atoms in the sample, producing various signals that contain information about the surface topography and composition of the sample. The electron beam is scanned in a raster scan pattern, and the position of the beam is combined with the
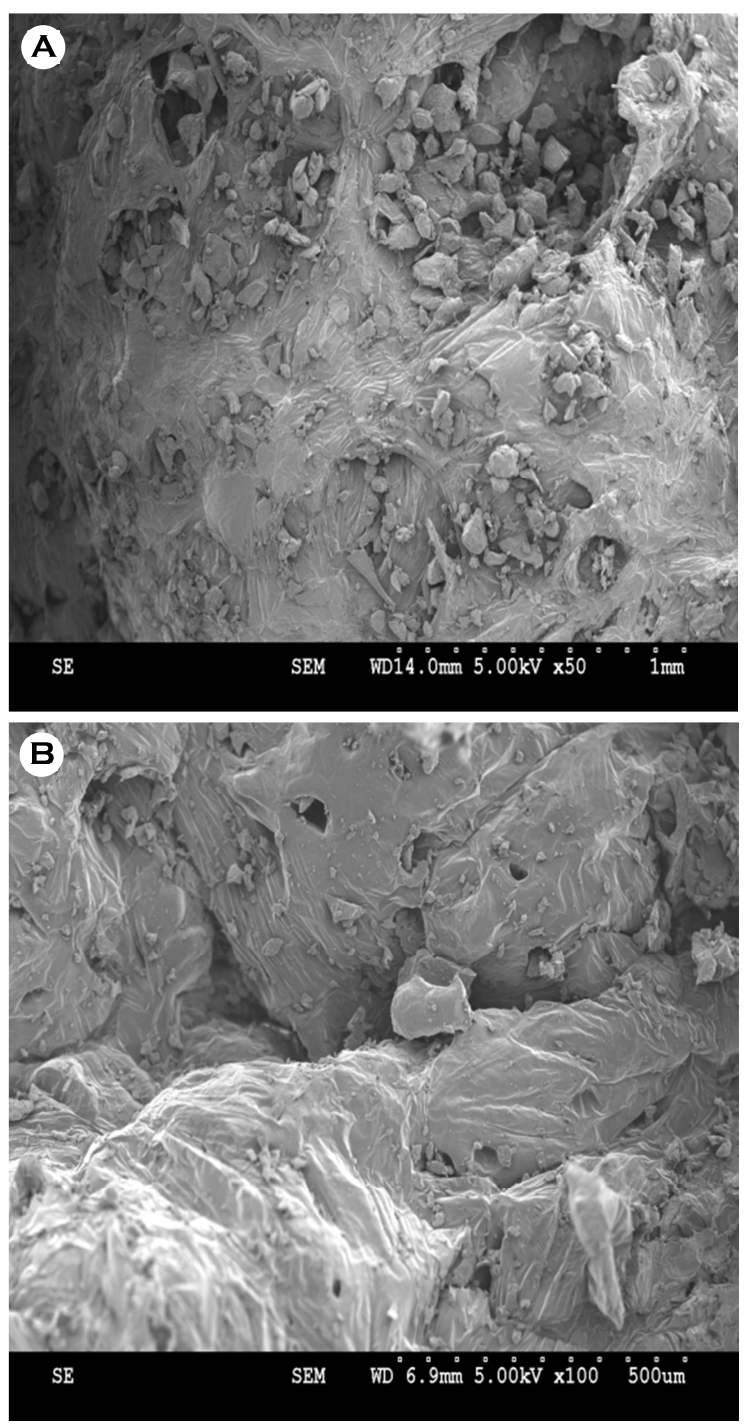

Fig. 3. SEM images of CA composites ((A) prepared by air drying (Left panel), (B) prepared by rotary evaporation (Right panel)).

detected signal to produce an image. The SEM images of the surface of the composite showed that the surface of CAsucrose ester composites prepared by the air drying method was rough and the particles were dispersed without clumping. In contrast, for the composites manufactured by rotary evaporation, the particles were clustered and the surface was smooth (Fig. 3).

\section{DISCUSSION}

Choline alphoscerate (CA) is a natural choline compound 
found in the brain. It is also a parasympathomimetic acetylcholine precursor, which may have potential for the treatment of Alzheimer's disease and other dementias. Alpha-GPC rapidly delivers choline to the brain across the blood-brain barrier and is a biosynthetic precursor of acetylcholine. In most countries, it is a non-prescription drug. Studies have investigated the efficacy of alpha-GPC for cognitive disorders, including stroke and Alzheimer's disease (Doggrell and Evans, 2003). A trial confirmed the therapeutic role of alphaGPC in the cognitive recovery of patients based on four measurement scales, three of which indicated statistical significance; commonly used daily doses were 300 to $1,200 \mathrm{mg}$ daily (Barbagallo et al., 1994). Industrially, CA is produced by the chemical or enzymatic deacylation of phosphatidylcholine-enriched soya phospholipids, followed by chromatographic purification. CA may also be derived in small amounts from highly purified soy lecithin, as well from purified sunflower lecithin. As CA is hygroscopic, this may cause gel capsules not fully packed with CA to dissolve. Proper storage methods need to be used with alpha-GPC, and include removal of all air from the container, double bagging with plastic bags rated for chemicals, and storage of bulk/ excess compound inside the freezer. It is important to note that the hygroscopic reaction does not cause degradation and the substance remains usable. Liquefied or gelled CA may also be indicative of poor storage and thus lead to an increased likelihood of actual degradation. In this study, we tried to solidify the liquid CA by using sucrose ester and characterized the CA-sucrose ester composites. Sucrose esters 570 and 1,170 were the most suitable vehicles for the preparation of the composite, and a 1:3 ratio was found to be the most suitable formulation. CA was successfully prepared as a solid powder by using sucrose ester; thus, it represents an effective solution for the limitations of conventional formulations.

\section{ACKNOWLEDGEMENT}

None.

\section{CONFLICTS OF INTEREST}

The authors declare that there is no conflict of interests regarding the publication this article.

\section{REFERENCES}

Barbagallo SG, Barbagallo M, Giordano M, Meli M, Panzarasa R. Alpha-Glycerophosphocholine in the mental recovery of cerebral ischemic attacks. An Italian multicenter clinical trial. Ann NY Acad Sci. 1994. 717: 253-269.

Cummings JL, Benson F. Subcortical dementia: Review of an emerging concept. Arch Neurol. 1984. 41: 874-879.

Doggrell SA, Evans S. Treatment of dementia with neurotransmission modulation. Expert Opin Investig Drugs. 2003. 12: 1633-1654.

Fossati T, Colombo M, Castiglioni C, Abbiati G. Determination of plasma choline by high-performance liquid chromatography with a postcolumn enzyme reactor and electrochemical detection. J Chromatogr B Biomed Appl. 1994. 656: 59-64.

Gatti G, Barzaghi N, Acuto G, Fossati T, Perucca E, Abbiatti G. A comparative study of free plasma choline levels following intramuscular administration of L- $\alpha$-glycerylphosphorylcholine and citicoline in normal volunteers. Int J Clin Pharmacol Ther. 1992. 30: 331-335.

Grossman H, Bergmann C, Parker S. Dementia: A brief review. Mt Sinai J Med. 2006. 73: 985-992.

Kidd PM. Alzheimer's disease, amnestic mild cognitive impairment, and age-associated memory impairment: current understanding and progress toward integrative prevention. Altern Med Rev. 2008. 13: 85-115.

Majahabulin S. Enzymatic synthesis of sugar fatty acid esters in organic solvent and in supercritical carbon dioxide and their antimicrobial activity. J Supercrit Fluids. 2008. 45: 338-345.

Moreno DJ. Cognitive improvement in mild to moderate Alzheimer's dementia after treatment with the acetylcholine precursor choline alfoscerate: a multicenter, double-blind, randomized, placebo-controlled trial. Clin. Ther. 2003. 25: 178-193.

Parnetti L, Mignini F, Tomassoni D, Traini E, Amenta F. Cholinergic precursors in the treatment of cognitive impairment of vascular origin: ineffective approaches or need for re-evaluation. J Neurol Sci. 2007. 257: 264-269.

Park SY, Choi SU, Lee KH, Yang JB, Cho, SW, Ro JE, Kim HG, Khadka P, Lee JH, Cho CW. Discriminative measurement and pharmacokinetic evaluation of choline alphoscerate against endogenous choline in human. Bull Korean Chem Soc. 2015. 36: 2089-2094.

Sheard NF, Tayek JA, Bistrian BR, Blackburn GL, Zeisel SH. 
Plasma choline concentration in humans fed parenterally. Am J Clin Nutr. 1986. 43: 219-224.

Sheard NF, Zeisel SH. An in vitro study of choline uptake by intestine from neonatal and adult rats. Pediatr Res. 1986. 20: 768-72.

Trainia E, Bramantib V, Amenta F. Choline alphoscerate (alphaglyceryl-phosphoryl-choline) an old choline-containing phospholipid with a still interesting profile as cognition enhancing agent. Curr Alzheimer Res. 2013. 10: 1070-1079.

https://doi.org/10.15616/BSL.2019.25.2.170

Cite this article as: Kim H, Cho SW. Preparation and Evaluation of Solid Composites Containing Choline Alphoscerate. Biomedical Science Letters. 2019. 25: 170 -176 . 